Pacific Journal of Mathematics

CHER CLASSES AND COHOMOLOGY FOR RANK 2
REFLEXIVE SHEAVES ON $\mathrm{P}^{3}$ 


\title{
CHERN CLASSES AND COHOMOLOGY FOR RANK 2 REFLEXIVE SHEAVES ON $\mathbf{P}^{3}$
}

\author{
MARgherita Roggero and PaOlo Valabrega
}

The paper shows that, if $F$ is a nonsplit rank 2 reflexive sheaf on $\mathbf{P}^{3}$, then the knowledge of the numbers $d_{n}=h^{2}(F(n))-h^{1}(F(n))$ gives an explicit algorithm to compute the Chern classes $c_{1}, c_{2}, c_{3}$ and the dimensions $h^{0}(F(n))$, for all $n$ (in particular the first integer $a$ such that the sheaf $F(a)$ has some nonzero section). If the sheaf is a vector bundle it is also proved that the knowledge of the numerical sequence $\left\{h^{1}(F(n))\right\}$ together with the first Chern class gives all the information as above. In some special cases, i.e. when $h^{1}(F(n)) \neq$ 0 for at most three values of $n$, an algorithm is also produced to compute the first Chern class from the sequence $\left\{h^{1}(F(n))\right\}$. Vector bundles with natural cohomology are also discussed.

It must be remarked that, if one knows not only the dimensions $h^{1}(F(n))$, for all $n$, but also the whole structure of the Rao-module $\bigoplus H^{1}(F(n))$, then the first Chern class $c_{1}$ is uniquely determined (as it is shown in a paper by $\mathbf{P}$. Rao).

n1. $F$ is a rank 2 nonsplit reflexive sheaf on $\mathbf{P}^{3}=\mathbf{P}$. Its Chern classes are $c_{1}, c_{2}, c_{3}$; if it is normalized, then $c_{1}=0$ or -1 . Once and for all $\left.h^{i}(F(n))=\operatorname{dim} F(n)\right), i=0,1,2,3$.

Now we give a list of well-known properties useful throughout the paper.

1. If $c_{1}(F)=c_{1}$, the associated normalized reflexive sheaf is defined as $F^{n}=F(\varepsilon)$ where

$$
\varepsilon= \begin{cases}\frac{c_{1}}{2} & \text { for } c_{1} \text { even } \\ -\frac{c_{1}+1}{2} & \text { for } c_{1} \text { odd }\end{cases}
$$

2. With every reflexive sheaf $F$ there are two associated numbers:

$$
\begin{gathered}
a=a(F)=\text { smallest integer } n \text { such that } h^{0}(F(n)) \neq 0, \\
a_{1}=a_{1}(F)=\text { smallest integer } n \geq a \text { such that } \\
\\
h^{0}(F(n))>h^{0}\left(O_{P}(n-a)\right) .
\end{gathered}
$$

Since $F$ is not split, then every general nonzero section of $F(a)$ gives rise to a zero locus which is necessarily a curve in $\mathbf{P}$ (see [H1], n. 1 and [H2], n.4); this is false for a split sheaf. The same is true for 
$F(n), n \geq a_{1}$ (see [GRV], Th. 0.1: it is stated for bundles, but it is easy to check it holds for any reflexive sheaf, exactly with the same proof: actually we shall use it only for bundles).

3. If a section of $F(n)$ gives rise to a curve, then $F$ and the ideal sheaf $I$ of the curve are connected by the following exact sequence (see [H1], n.1 and [H2], n.4):

$$
0 \rightarrow O_{P} \rightarrow F(n) \rightarrow \mathbf{I}\left(2 n+c_{1}\right) \rightarrow 0 .
$$

It follows that, if in addition $F$ is normalized, then $h^{0}(F(n))>1$ implies that $n>0$, because $n \leq 0$ means that the curve lies on a surface of degree $2 n+c_{1} \leq 0$, which is absurd.

In particular $a_{1}>0$ and $h^{0}(F(a))=1$ if $a \leq 0$, because in both cases there is a curve (see 2 above).

4. The Euler-Poincare function of $F$ is

$$
\begin{aligned}
\chi(F(n))= & (n+3)(n+2)(n+1) / 3+c_{1}(n+2)(n+1) / 2 \\
& -(n+2) c_{2}+\left(c_{3}-c_{1} c_{2}\right) / 2 .
\end{aligned}
$$

5. The normalized sheaf $F$ is stable if $a(F)=a>0$, properly semistable if $a=c_{1}=0$, unstable otherwise; a general sheaf is stable, properly semistable, unstable according to the corresponding property for the normalized sheaf $F^{n}$.

6. If $F$ is a nonsplit vector bundle, then $h^{1}(F(n)) \neq 0$ for some $n$ (Horrocks' theorem: see [BH], n.5, Lemma 1). Actually it is true: if $c_{1}$ is even, then $h^{1}(F(\varepsilon-1)) \neq 0$; if $c_{1}$ is odd, then $h^{1}(F(n)) \neq 0$ for $n=\varepsilon-1, \varepsilon, \varepsilon+1$. This depends upon [CV1], Theorems 1.3 and 1.6; in fact it is enough to consider, for $n \gg 0$, a smooth irreducible curve $C$ zero locus of a section of $F(n)$; then, by $(*)$ above $h^{1}(F(m))=$ $h^{1}\left(\mathbf{I}\left(n+m+c_{1}\right)\right.$, for all $m$.

Moreover $h^{1}(F(n)) \neq 0$ for all $n$ such that $a-2 \leq n \leq a_{1}-2$ ([CV2], Cor. 8).

7. For a reflexive sheaf $F$ the condition $h^{1}(F(n))=0$ for every $n$ is far from forcing the sheaf to split; in fact every locally complete intersection curve $C$ is zero locus of a section of some reflexive sheaf $F$ ([H2], Th. 4.1), but $C$ may as well be arithmetically normal, which implies (by $(*)$ above): $h^{1}(F(n))=h^{1}(\mathbf{I}(n))=0$ for every $n$. If $C$ is not a complete intersection, then $F$ cannot split ([H1], Cor. 1.2):

8. A condition that forces a reflexive sheaf to be a bundle is given in [R], Theorem 2.3: the normalized sheaf $F$ is a vector bundle if and only if $h^{2}(F(p))=0$ for some $p \leq-2$; for a general sheaf this condition becomes: $h^{2}(F(p))=0$ for some $p \leq-2+\varepsilon$. 
If

$$
h^{2}(F(p))=0 \text { for } \begin{cases}p=\varepsilon-3 & \text { if } c_{1} \text { is even } \\ p=\varepsilon-4 \text { or } \varepsilon-3 \text { or } \varepsilon-2 & \text { if } c_{1} \text { is odd }\end{cases}
$$

then the sheaf is a split bundle ([R], Cor. 3.2).

9. The dual $F^{\vee}$ of $F$ is the reflexive sheaf $F\left(-c_{1}\right)$ ([H1], \#2 and [H2], Prop. 1.10: recall that the first Chern class can be defined through the determinant itself). Moreover, if $F$ is a vector bundle, Serre's duality says that $h^{i}(F(n))=h^{3-i}\left(F\left(-n-c_{1}-4\right)\right)$ for every $n$ and $i=0,1$. If $F$ is an arbitrary reflexive sheaf, then the above equality holds only for $i=0$ ([H2], Prop. 2.5), while for $i=1$ we have the following exact sequence

$$
\begin{aligned}
(* *) \quad 0 & \rightarrow H^{1}\left(F\left(-n-c_{1}-4\right)\right) \rightarrow H^{2}(F(n)) \rightarrow H^{0} \operatorname{Ext}^{1}\left(F(n), O_{\mathbf{P}}(-4)\right) \\
& \rightarrow H^{2}\left(F\left(-n-c_{1}-4\right)\right) \rightarrow H^{1}(F(n)) \rightarrow 0
\end{aligned}
$$

together with the equality: $h^{0} \widetilde{\mathrm{Ext}}^{1}\left(F(n), O_{\mathbf{P}}(-4)\right)=c_{3}$ (see [H2], Prop. 1.10, Th. 2.5, claim and proof of Prop. 2.6, Cor. 2.2: the sequence $(* *)$ is given with duals but $\left.F^{\vee}=F\left(-c_{1}\right)\right)$.

For a reflexive sheaf, we introduce, for every integer $n$ in $\mathbf{Z}$, the number $d_{n}=d_{n}(F)=h^{2}(F(n))-h^{1}(F(n)$ ) (see also [BH], n.3). For $n \ll 0, d_{n}(F)=h^{2}(F(n))$, because $h^{1}(F(n))=0$. If $F$ is a vector bundle, then $d_{n}=h^{1}\left(F\left(-n-c_{1}-4\right)\right)-h^{1}(F(n))$, because of Serre's duality (not true in general for a reflexive sheaf); therefore, $d_{n}(F)=0$ both for $n \ll 0$ and for $n \gg 0$.

We start with the following key lemma:

LEMMA 1. Let $F$ be a nonsplit reflexive sheaf.

(i) if $F$ is normalized and $n \leq 0$, then $h^{0}(F(n))=h^{0}\left(O_{\mathbf{P}}(n-a)\right)$;

(ii) if $F$ is general and $n+a+c_{1} \leq 0, h^{0}(F(n))=h^{0}\left(O_{\mathbf{P}}(n-a)\right)$;

(iii) if $F$ is general and $n+a+c_{1}>0$, then $h^{0}(F(n))=\chi(F(n))-$ $d_{n}$.

Proof. (i) if $a>0$, then $n<a$, so both members are 0 . If $a \leq 0$, take a curve $Y$ corresponding to the sheaf $F(a)$, getting the exact sequence (see above)

$$
0 \rightarrow O_{\mathbf{P}} \rightarrow F(a) \rightarrow \mathbf{I}_{Y}\left(2 a+c_{1}\right) \rightarrow 0
$$


and also (twisting by $n-a$ )

$$
0 \mapsto O_{\mathbf{P}}(n-a) \mapsto F(n) \mapsto \mathbf{I}_{Y}\left(n+a+c_{1}\right) \mapsto 0 .
$$

It is now enough to remark (because $c_{1} \leq 0$ for a normalized sheaf) that $n+a+c_{1} \leq 0$, which implies that no surface of degree $n+a+c_{1}$ contains $Y$.

(ii) The same exact sequence as above works: now $c_{1}$ is arbitrary but $n+a+c_{1} \leq 0$ by hypothesis.

(iii) We have: $h^{0}(F(n))=\chi(F(n))-d_{n}+h^{0}\left(F\left(-n-c_{1}-4\right)\right)$, but the last term is 0 because $-n-c_{1}-4<a$ by hypothesis.

We want to show that the knowledge of the numbers $d_{n}$ gives some information about $F$, i.e. about the Chern classes, the number $a(F)$, the property of being stable or unstable,... .

First of all we give a criterion to decide whether $F$ is stable or not, based on the knowledge of the numbers $d_{n}$ 's and of $c_{1}$, hence of the normalized sheaf $F^{n}=F(\varepsilon)$ (see above). We recall that, for every $n$,

$$
\begin{aligned}
d_{n}(F)= & h^{2}(F(n))-h^{1}(F(n))=h^{2}(F(\varepsilon)(n-\varepsilon)) \\
& -h^{1}(F(\varepsilon)(n-\varepsilon))=d_{n-\varepsilon}(F(\varepsilon))=d_{n-\varepsilon}\left(F^{n}\right) .
\end{aligned}
$$

Proposition 1. Let $F$ be a nonsplit reflexive sheaf and $n, m$ two integers. Then

$$
\begin{aligned}
n d_{\varepsilon} & +m d_{-1+\varepsilon}-(2 m+3 n) d_{-2+\varepsilon}+(m+2 n) d_{-3+\varepsilon} \\
& = \begin{cases}2 n+(m+3 n)\left(c_{1}+2 \varepsilon\right) & \text { (stable sheaf }) \\
n & \text { (properly semistable sheaf) } \\
0 & \text { (unstable sheaf) }\end{cases}
\end{aligned}
$$

Proof. First we prove the claim when $F$ is normalized, i.e. when $\varepsilon=0$. By Lemma 1 we have, for $h \leq 0$ :

$$
h^{0}(F(h))=h^{0} O_{\mathbf{P}}(h-a) .
$$

Moreover, if $h \geq-3$, then $h^{3}(F(h))=h^{0}\left(F\left(-h-c_{1}-4\right)\right)=$ $h^{0} O_{\mathbf{P}}\left(-h-c_{1}-4-a\right)$ because $-h-c_{1}-4 \leq 0$. Substitute now $d_{h}$ with $\chi F(h)-h^{0} F(h)+h^{3} F(h)$ in $n d_{0}+m d_{-1}-(2 m+3 n) d_{-2}+(m+2 n) d_{-3}$ and use Lemma 1 and duality; if we put: $\varphi(n)=n+1$ for $n \geq 0$, $\varphi(n)=0$ for $n<0$, we get:

$$
\begin{gathered}
2 n+(3 n+m) c_{1}+n\left(-\varphi(-a)-2 \varphi(-a-1)+2 \varphi\left(-a-1-c_{1}\right)\right. \\
\left.+\varphi\left(-a-2-c_{1}\right)\right) \\
+m\left(\varphi\left(-a-c_{1}-1\right)-\varphi(-a-1)\right)
\end{gathered}
$$


Therefore, if $F$ is stable $(a>0)$ the result is $2 n+(3 n+m) c_{1}$; if it is property semistable ( $a=0$ and $c_{1}=0$ ) the result is $n$; if it is unstable the result is 0 .

Assume now that $F$ is general; then it is enough to remark that $d_{n+\varepsilon}(F)=d_{n}\left(F^{n}\right)$ for all $n$ and moreover $c_{1}+2 \varepsilon=c_{1}(F(\varepsilon))([\mathbf{H} 2]$, Cor. 2.2).

Proposition 2. Let $F$ be a nonsplit rank 2 reflexive sheaf on $\mathbf{P}$. If it is stable then $a-\varepsilon$ is the smallest positive integer $n$ such that

$$
d_{n+\varepsilon}-(n+2) d_{-1+\varepsilon}+(n+1) d_{-2+\varepsilon} \neq 2\left(\begin{array}{c}
n+3 \\
3
\end{array}\right)+\left(c_{1}+2 \varepsilon\right)\left(\begin{array}{c}
n+2 \\
3
\end{array}\right) \text {. }
$$

If it is unstable then $-a+\varepsilon-c_{1}$ is the smallest positive integer $n$ such that $d_{n+\varepsilon}-(n+2) d_{-1+\varepsilon}+(n+1) d_{-2+\varepsilon} \neq 0$.

Proof. First assume that $F$ is normalized $(\varepsilon=0)$, that $a>0$ and $c_{1}=0$. Then we have (for any $\left.n \geq 0\right): d_{n}=\chi(F(n))-h^{0}(F(n))$, by Lemma 1, (iii); therefore

$$
\begin{aligned}
d_{n}- & (n+2) d_{-1}+(n+1) d_{-2} \\
& =\chi(F(n))-h^{0}(F(n))-(n+2)(\chi(F(-1))) \\
& =n^{3} / 3+2 n^{2}+11 n / 3+2-(n+2) c_{2}-h^{0}(F(n))-(n+2)\left(-c_{2}\right) \\
& =2\left(\begin{array}{c}
n+3 \\
3
\end{array}\right)-h^{0}(F(n))
\end{aligned}
$$

which gives the result because the sheaf has no nonzero section up to $n=a-1$ and at least one nonzero section for $n=a$.

If $c_{1}=0$ and $a<0$, then, for all $n$ such that $0 \leq n \leq-a$, we have:

$$
\begin{aligned}
d_{n}- & (n+2) d_{-1}+(n+1) d_{-2} \\
= & \chi(F(n))-h^{0}(F(n))+h^{0}(F(-n-4)) \\
& -(n+2)\left(\chi(F(-1))-h^{0}(F(-1))+h^{0}(F(-3))\right) .
\end{aligned}
$$

Since $0 \leq n \leq-a$, then we are able to use Lemma 1, (i) and (ii), to see that the second member is simply

$$
\begin{aligned}
2\left(\begin{array}{c}
n+3 \\
3
\end{array}\right) & -\left(\begin{array}{c}
n-a+3 \\
3
\end{array}\right)+h^{0} O_{\mathbf{P}}(-n-a-4) \\
& +(n+2)\left(h^{0} O_{\mathbf{P}}(-3-a)-h^{0} O_{\mathbf{P}}(-1-a)\right)
\end{aligned}
$$

which is easily seen to be 0 if $n<-a$ and 1 if $n=-a$.

The case $c_{1}=-1$ can be dealt with using very similar arguments. 
Now pass to $F$ arbitrary: it is enough to recall that $d_{n}(F)=$ $d_{n-\varepsilon}(F(\varepsilon))$ and $c_{1}(F(\varepsilon))=c_{1}+2 \varepsilon$ ([H2], Cor. 2.2).

REMARK 1. It is not evident at all that the same sequence $\left\{d_{n}\right\}$ for two different reflexive sheaves gives rise to the same $a$; actually this is true, because the $d_{n}$ 's determine also $c_{1}$, as it will be seen in Theorem 1 .

Corollary 1. A reflexive sheaf $F$ splits if and only if $d_{n}=0$ for all $n$.

Proof. If $F$ is split, then it is well known that the intermediate cohomology vanishes.

Conversely, assume that all the $d_{n}$ 's vanish and $F$ does not split; then, by Prop. 1, $F$ must be an unstable sheaf; now use Prop. 2 to see that $d_{n} \neq 0$ either for $n=-a+2 \varepsilon-c_{1}$ or for $n=-2+\varepsilon$ or for $n=-1+\varepsilon$. This is a contradiction.

REMARK 2 . The same result could be proved using the nonsplitting criteria of $[\mathbf{R}]$ (see 8 above).

Proposition 3. Let $F$ be a nonsplit rank 2 reflexive sheaf on $\mathbf{P}$. Then we have:

(i) if $F$ is stable or properly semistable, then $c_{2}=-\varepsilon^{2}-c_{1} \varepsilon+$ $d_{-2+\varepsilon}-d_{-1+\varepsilon}$;

(ii) if $F$ is unstable, then $c_{2}=-\varepsilon^{2}-c_{1} \varepsilon+d_{-2+\varepsilon}-d_{-1+\varepsilon}-$ $(a-\varepsilon)^{2}-(a-\varepsilon)\left(c_{1}+2 \varepsilon\right)=-a^{2}-a c_{1}+d_{-2+\varepsilon}-d_{-1-\varepsilon}$.

Proof. First we assume that $F$ is normalized $(\varepsilon=0)$. If $a \geq 0$, then $c_{2}=\chi(F(-2))-\chi(F(-1))=d_{-2}-d_{-1}$. If $a<0$, then we have:

$$
\begin{aligned}
d_{-2}-d_{-1}= & \chi(F(-2))-\chi(F(-1))-h^{0}(F(-2))+h^{0}\left(F\left(-2-c_{1}\right)\right) \\
& +h^{0}\left(F(-1)-h^{0}\left(F\left(-3-c_{1}\right)\right)\right. \\
= & c_{2}-h^{0}\left(O_{\mathbf{P}}(-2-a)\right)+h^{0}\left(O_{\mathbf{P}}\left(-2-a-c_{1}\right)\right) \\
& +h^{0}\left(O_{\mathbf{P}}(-a-1)\right)-h^{0}\left(O_{\mathbf{P}}\left(-a-3-c_{1}\right)\right) \\
= & c_{2}+a^{2}+a c_{1}+\left(c_{1}^{2}+c_{1}\right) / 2=c_{2}+a^{2}+a c_{1} .
\end{aligned}
$$

If the sheaf is arbitrary we pass to $F(\varepsilon)$ and recall that $c_{2}(F(\varepsilon))=$ $c_{2}(F)+c_{1}(F) \varepsilon+\varepsilon^{2}([\mathbf{H} 2]$, Cor. 2.2).

REMARK 3. The previous results show that, if we have the sequence $\left\{d_{n}\right\}$, for all $n$, and $c_{1}$ (hence $\varepsilon$ ), then there is an algorithm to compute explicitly both $a$ and $c_{2}$. 
LEMMA 2. Let $F$ be a nonsplit rank 2 reflexive sheaf; then

(i) $d_{n}=0$ for all $n \gg 0$;

(ii) $d_{n}=c_{3}$ for all $n \ll 0$.

Proof. The intermediate cohomology for any coherent sheaf is 0 for $n \gg 0$; therefore (i) is obvious. For (ii) we remark that the first cohomology module is finite, hence 0 for $n \ll 0$ and then use the exact sequence $(* *)$ of $\mathrm{n} .1$, which for $n \ll 0$ has only two nonzero terms $\left(h^{2}(F(n))\right.$ and $\left.c_{3}\right)$.

By the previous lemma and Corollary 1 every nonsplit reflexive sheaf $F$ has two associated numbers (depending upon $\left\{d_{n}\right\}$ : if all the $d_{n}$ 's are 0 , then they are not defined):

$$
\begin{aligned}
& p=p(F)=\min \left\{n / d_{m}=0 \forall m \geq n\right\} ; \\
& q=q(F)=\max \left\{n / d_{m}=c_{3} \forall m \leq n\right\} .
\end{aligned}
$$

Now we show some relations between the $d_{n}$ 's, the Chern classes, $a(F)$ and $h^{0}(F(n))$, for all $n$. Actually we see that, if the sequence $\left\{d_{n}\right\}$ is known for all $n$, then all the numerical characters are explicitly computable from it.

THEOREM 1. Let $F$ be a nonsplit rank 2 reflexive sheaf. Then we have:

(i) $c_{3}=d_{q}$;

(ii) $c_{1}=-p-q-4$;

(iii) $c_{2}$ is uniquely determined by $c_{1}$ and $\left\{d_{n}\right\}$ as in Proposition 3;

(iv) $a$ is uniquely determined by $c_{1}$ and $\left\{d_{n}\right\}$ as in Proposition 2;

(v) $h^{0}(F(n))$ is uniquely determined by $c_{1}$ and $\left\{d_{n}\right\}$ as in Lemma 1, (ii) and (iii).

Proof. (i) follows from Lemma 2.

For (ii) we observe that, by the above exact sequence (**) of n.1, we have:

therefore

$$
c_{3}=d_{n}+d_{-n-c_{1}-4}
$$

$$
d_{-q-c_{1}-4+r}=c_{3}-d_{q-r}= \begin{cases}0 & \text { for } r \leq 0 \\ t \neq 0 & \text { for } r>0\end{cases}
$$

which means (by the definition itself) that $p=-q-c_{1}-4$.

For (iii), (iv), (v), we remark that, by (ii), $p$ and $q$ determine $c_{1}$, hence also $\varepsilon$. 
REMARK 4. The equality

$$
c_{3}=d_{n}+d_{-n-c_{1}-4}
$$

shows that the pairs $\left(n, d_{n}\right)$ are symmetric with respect to $\left(-\left(c_{1}+4\right) / 2, c_{3} / 2\right)$.

For a rank 2 nonsplit vector bundle we know that, by Serre's duality, $h^{2}(F(n))=h^{1}\left(F\left(-n-c_{1}-4\right)\right)$; moreover $c_{3}=0$ ([H2], Prop. 2.6). So the previous result becomes as follows:

COROLlaRY 2. Let $F$ be a nonsplit rank 2 vector bundle over $\mathbf{P}$. Then the sequence $\left\{h^{1}(F(n))\right\}$ and the Chern class $c_{1}$ determine uniquely the sequence $\left\{d_{n}\right\}$, hence $c_{2}$, a and $h^{0}(F(n))$, for all $n$.

REMARK 5. In [GRV] these results were already obtained, but only for a bundle $G$ having the same sequence $\left\{h^{1}(G(n))\right\}$ as a bundle associated to a skew union of complete intersection.

n.2. Corollary 2 in n.1 leads us to raise the following question: is it enough to know the sequence $\left\{h^{1}(F(n))\right\}$, all $n$, in order to get also $c_{1}$ ? We do not know in general, but we discuss some examples and partial results.

EXAMPLE 1. If there is $m$ such that $h^{1}(F(n))=0$ for $n \neq m$, $h^{1}(F(m)) \neq 0$, then the sheaf $F(m+1)$ is a nullcorrelation bundle. In fact, if the normalized bundle $F(\varepsilon)=E$ is not stable, that is, $a(E) \leq 0$, then $h^{1}(E(n)) \neq 0$ for $a-2 \leq n \leq a_{1}-2$ (see n.1,6; see also [S], Th. 3.1 and Prop. 3.2), which includes at least 2 values.

Therefore the bundle is stable. In this case either the 1-cohomology vanishes at 2 values of $n$ or it is a nullcorrelation bundle (see [CV1], Prop. 4).

Proposition 4. Let $F$ be a rank 2 vector bundle such that $h^{1}(F(n))$ does not vanish exactly at 2 values $n=m$ and $n=p>m$; then we have:

the bundle is stable;

$$
\begin{aligned}
& p=m+1 ; \\
& c_{1}=-2 m-2 ; \\
& c_{2}=2+(m+1)^{2} ; \\
& h^{1}(F(n))= \begin{cases}2 & \text { for } n=m, \\
2 & \text { for } n=m+1\end{cases}
\end{aligned}
$$


(the same dimensions as a twist of the bundle associated to 3 skew lines).

Proof. by [CV1], Th. 1.3, 1.5 and 1.6 and [CV2], Cor. 8 (see above, n.1,6), the hypothesis implies that the bundle has even $c_{1}$, i.e. the normalized bundle $F^{n}=E$ has $c_{1}=0$, the 1-cohomology cannot vanish exactly at -1 and at 0 and $a(E)-2 \geq-1$, which means that the bundle is stable. Now we are able to compute explicitly such a cohomology at both levels where it does not vanish. Working on the normalized bundle $E$, with $c_{1}=0, c_{2}>1$ (by [H1], Lemma 3.2), we have:

$$
\begin{aligned}
\chi(E) & =-h^{1}(E)=2-2 c_{2}<0 ; \\
\chi(E(-1)) & =-h^{1}(E(-1))=-c_{2} ; \\
\chi(E(1)) & =h^{0}(E(1))=8-3 c_{2}>0 .
\end{aligned}
$$

Therefore $c_{2}$ must be either 1 or 2 , and hence $c_{2}=2$.

Now we go back to the original bundle and find that the first nonvanishing level must be $m=-c_{1} / 2-1$, whence the expected value $c_{1}=2(-m-1)$. The claim about $c_{2}$ follows from the general formula connecting the second Chern class of a bundle and of a twist.

Proposition 5. Let $F$ be a nonsplit rank 2 vector bundle such that $c_{1}=0$ and moreover $h^{1}(F(n))$ does not vanish exactly for three values of $n$. Then:

(i) $h^{1}(F(n)) \neq 0$ for $n=-1,0,1$;

(ii) $F$ is stable;

(iii) $3 \leq c_{2} \leq 5$ and moreover

$$
h^{1}(F(n))= \begin{cases}c_{2} & \text { for } n=-1, \\ 2 c_{2}-2 & \text { for } n=0, \\ t & \text { for } n=1,\end{cases}
$$

where $t=3 c_{2}-8+h^{0}(F(1))$ and $h^{0}(F(1)) \leq 5-c_{2}$.

Proof. By [CV1], Prop. 15 (see n.1, 6), the cohomology is not 0 at $n=-1$ and at $n=0$ (to avoid a nullcorrelation bundle, with only one non vanishing group). Moreover, the cohomology does not vanish between $a-2$ and $a_{1}-2$; since $a_{1}>0$ (Remark 1), then $a \geq 0$. Now put: $c_{2}(F)=c$. If $a=0$ the three vanishing groups correspond to $n=-2,-1,0$. Then we have: $\chi(F(1))=h^{0}(F(1))=8-3 c \geq 4$, which means either $c=0$ or $c=1$; but both cases are absurd, because $F$ has a section which, being the first one, gives rise to a curve of degree $c$ (see [H1], Lemma 1.3). 
At last $a>0$ and the bundle is stable. Now we want to show that the third nonvanishing group corresponds exactly to $n=1$. In fact, assume that $h^{1}(F(1))=0$; then we have: $\chi(F(1))=8-3 c=$ $h^{0}(F(1))+h^{1}(F(-5)) \geq 0$, which implies $c \leq 2$ : but $c \leq 1$ is excluded because the bundle is stable ([H1], Lemma 3.2) and $c=2$ is excluded by [H1], Lemma 9.4 (because there should be only two nonvanishing groups). Hence the three nonvanishing groups correspond to $n=-1,0,1$ and the dimensions can be computed by $\chi(F(-1))=$ $-c, \chi(F)=2-2 c, \chi(F(1))=8-3 c=h^{0}(F(1))-h^{1}(F(1))$. Вy $\chi(F(2))=h^{0}(F(2))=20-4 c$ we see that $20-4 c \geq 0$, hence $c \leq 5$; moreover $c \geq 3$, because 2 and 1 are excluded by [H1], Lemma 3.2 and Lemma 9.4)

For the value of $t$ it is enough to observe that $h^{0}(F(2))$ is 0 for $c=5$ and so $h^{0}(F(1))=0$; if $c=4$, then $h^{0}(F(2))=4$. Hence $F(1)$ has at most one nonzero section; if $c=3$, then $h^{0}(F(2))=8$ and $F(1)$ has at most two independent sections.

Proposition 6. Let $F$ be a nonsplit rank 2 vector bundle such that $c_{1}=-1$ and moreover $h^{1}(F(n))$ does not vanish exactly at three values of $n$. Then

(i) $h^{1}(F(n)) \neq 0$ for $n=-1,0,1$;

(ii) $c_{2}=2$;

(iii) $F$ is stable;

(iv)

$$
h^{1}(F(n))= \begin{cases}1 & \text { for } n=-1 \\ 2 & \text { for } n=0 \\ 1 & \text { for } n=1\end{cases}
$$

Proof. First of all put: $c=c_{2}$. By [CV1], Th. 1.6 see n.1, 6), the three nonvanishing groups must correspond to $n=-1,0,1$; therefore $a-2 \geq-2$ and $a \geq 0$ (see n.1,6). If $a=0$, then, because $\chi(F(2))=h^{0}(F(2))=14-7 c / 2 \geq 10$, we see that $c<8 / 7$. Hence $c=0$ (because $c$ must be odd: [H1], Cor. 2.2); but this is absurd because it is the degree of a curve, zero locus of a section of the bundle. Hence $a>0$ and the bundle is stable. If $a=1$ and $h^{0}(F(1))>1$, then there should exist a curve, zero locus of a section of $F(1)$, lying on a plane, which is absurd because it cannot be complete intersection as the bundle is not split ([H1], Cor. 1.2). Therefore either $a \geq 2$ or $a=1$ and there is only one independent section for $n=a=1$. Now we observe that $\chi(F(1))=5-5 c / 2, \chi(F(-2))=c / 2=h^{1}(F(-1))$, $\chi(F)=1-3 c / 2=-h^{1}(F), \chi(F(2))=h^{0}(F(2))=14-7 c / 2$; hence 
we see that $c \leq 4$ and therefore either $c=2$ or $c=4$ ( $c$ being even for an odd first Chern class). If $c=2$, then we have the following cohomology: $h^{1}(F(-1))=1, h^{1}(F)=2, h^{1}(F(1))=1 \quad(F(2)$ gives rise to two skew conics). The case $c=4$ is not allowed because it implies $a \geq 3$ and hence natural cohomology, which is excluded by [HH], Ex. 1.6.3.

THEOREM 2. Let $F$ be a nonsplit rank 2 vector bundle such that $h^{1}(F(n))$ does not vanish exactly at 3 values of $n$; then we have:

(i) the 1-cohomology does not vanish at 3 consecutive values $m$, $m+1, m+2$;

(ii) $F$ is stable;

(iii) setting $(a, b, c)=\left(h^{1}(F(m)), h^{1}(F(m+1)), h^{1}(F(m+2))\right)$ : either $(a, b, c)=(1,2,1)$ and then $c_{1}=-2 m-3$ or $(a, b)=$ $(h, 2 h-2)$, with $3 \leq h \leq 5$, and then $c_{1}=-2 m-2,3 h-8 \leq 2 h-3$. Furthermore, each of these seven cases actually occurs.

Proof. (i) The three values are consecutive for a normalized bundle, by Propositions 5 and 6; hence for every bundle.

(ii) The property is true for a normalized bundle (Propositions 5 and 6$)$, hence for all bundles.

(iii) The case $(a, b, c)=(1,2,1)$ is considered in Proposition 6 , and corresponds to an odd $c_{1}$, while the case $(a, b)=(h, 2 h-2)$ is considered in Proposition 5 and corresponds to an even $c_{1}$.

If $c_{1}$ is even, then the normalized bundle $F\left(-c_{1} / 2\right)$ has nonvanishing cohomology at $n=-1,0,1$ by Proposition 5; therefore we see that $m=-c_{1} / 2-1$. If $c_{1}$ is odd, then the normalized bundle $F\left(-\left(c_{1}+1\right) / 2\right)$ has nonvanishing cohomology at $n=-1,0,1$ by Proposition 6; therefore $m=-\left(c_{1}+1\right) / 2-1$.

Now we see that all the seven cases occur.

For $c_{1}=0$, the three values for $c_{2}$ are $3,4,5$; then

-if $c_{2}=3$, the sequences $(3,4,2)$ and $(3,4,3)$ for the 1-cohomology correspond to 4 skew lines lying either on one or no quadric surface;

-if $c_{2}=3$, the sequence $(3,4,1)$ corresponds to an instanton bundle with $a \leq 2$ ([H1], Th. 8.2 and [HH], examples of n.1);

-if $c_{2}=4,5$ skew lines give rise to the sequence $(4,6,5)$, but also the sequence $(4,6,4)$ is allowed ([HH], n.1);

-if $c_{2}=5$, the sequence $(5,8,7)$ exists and corresponds to a bundle with natural cohomology (again [HH], n.1).

As long as $c_{1}$ is odd, such a bundle corresponds to a skew union of two conics ([HH], Ex. 1.6.2). 
REMARK 6 . If the bundle has exactly 4 nonvanishing groups, then it is still possible to describe everything (the four values are consecutive, $c_{1}$ even and odd are not allowed together and can be explicitly computed by the position of the nonvanishing cohomology,... ), like in the previous theorem, but it is required, beside all the previous machinery, also the classification of stable bundles with $c_{1}=-1$ and $c_{2}=2$ (see [HS]).

For five nonvanishing groups there is a case that cannot be easily excluded; hence for $n \geq 5$ the difficulty increases, because of the lack of classification for normalized bundles. Therefore our technique cannot work for arbitrary $n$ (even if it is not excluded that some extra $n$ can be dealt with).

EXAMPLE 2. Let $F$ be a vector bundle with natural cohomology ([HH]) and $c_{1}(F)=-1$. Then $h^{1}(F(n)) \neq 0$ for $n=-1,0,1$ by [CV1], Th. 1.6 (n.1, 6). Therefore $h^{2}(F(-1))=h^{0}(F(-1))=$ $h^{2}(F)=h^{0}(F)=0$, because of the natural cohomology; hence $F$ is stable $(a \geq 1)$. Moreover $h^{2}(F(-1))=0=h^{1}(F(-2))$ implies that $F$ is an instanton bundle, which means that $h^{1}(F(n))=0$ for all $n \leq-2$ ([HH], Prop. 1.4). We have also:

$$
\begin{aligned}
h^{1}(F(-1)) & =-\chi(F(-1))=c_{2}(F) / 2, \\
h^{1}(F) & =-\chi(F)=3 c_{2}(F) / 2-1 .
\end{aligned}
$$

Now let $G$ be any bundle such that $c_{1}(G)=0$ and $h^{1}(G(n))=$ $h^{1}(F(n))$ for all $n$. By Proposition 2, with $n=1, m=0$, we have:

$$
\begin{aligned}
2 h^{1}(G(-1))-h^{1}(G)=2 h^{1}(F(-1))-h^{1}(F)=1-c_{2}(F) / 2 \\
\quad= \begin{cases}2 & (G \text { stable }), \\
1 & (G \text { properly semistable }), \\
0 & (G \text { unstable }) .\end{cases}
\end{aligned}
$$

So $c_{2}(F)=2$, because $F$ is stable (hence $c_{2}>0$ by [H1], Lemma 3.4). This implies that $h^{1}(F(-1))=1, h^{1}(F)=2$, while $h^{1}(F(1))=$ $-\chi(F(1))=-5+5 c_{2}(F) / 2=0$, which is absurd (n.1,6).

This means that no bundle $G$ with $c_{1}(G)=0$ can have $h^{1}(G(n))$ $h^{1}(F(n))$, for all $n$.

Using the same techniques as above one can show that, if $F$ is a bundle with natural cohomology and $c_{1}(F)=0$, then no bundle $G$ with $c_{1}(G)=-1$ can have $h^{1}(G(n))=h^{1}(F(n))$, for all $n$. 
EXAMPLE 3. It is possible that two normalized vector bundles have the same positive spectrum (see [H2], n.7), hence the same dimensions of the first cohomology groups for every $n \leq 0$, but different first Chern classes. For instance $([\mathbf{H 2}]$, n.8) take the skew union $Y$ of a plane cubic and a complete intersection of two quadrics, which gives rise to a bundle $F$; then $E=F(-2)$ has first Chern class 0 and spectrum $(-1,0,1)$. If we take the skew union $X$ of a plane quartic and the complete intersection of a cubic and a quadric, then $X$ gives rise to a vector bundle which, twisted by -3 , has first Chern class -1 and has spectrum $(-2,-1,0,1)$. It is easy to see that the first cohomology modules have different dimensions when $n>0$.

\section{REFERENCES}

[BH] W. Barth and K. Hulek, Monads and moduli of vector bundles, Manuscripta Math., 25 (1978), 323-347.

[CV1] L. Chiantini and P. Valabrega, Subcanonical curves and complete intersections in projective 3-space Ann. Mat. Pura Appl., 138 (1984), 309-330.

[CV2] - On some properties of subcanonical curves and unstable bundles, Comm. Algebra, 15 (9) (1987), 1877-1887.

[GRV] A. Geramita, M. Roggero, and P. Valabrega, Subcanonical curves with the same postulation as $q$ skew complete intersections in projective 3-space, (to appear in Rend. Univ. Pol. Milano).

[H1] R. Hartshorne, Stable vector bundle of rank 2 on $\mathbf{P}^{3}$, Math. Ann., 238 (1978), 229-280

[H2] - Stable reflexive sheaves, Math. Ann., 254 (1980), 121-176.

[HH] R. Hartshorne and A. Hirshowitz, Cohomology of a general instanton bundle, Ann. Sci. École Norm., 15 (1982), 365-390.

[HS] R. Hartshorne and I. Sols, Stable rank 2 vector bundles on $\mathbf{P}^{3}$, J. Reine Angew. Math., 325 (1981), 145-152.

[Ra] P. Rao, A note on cohomology modules of rank two bundles, (preprint)

[R] M. Roggero, On the rank 2 reflexive sheaves and the subcanonical curves in $\mathbf{P}^{3}$, Comm. Algebra, 16 (9) (1988), 1779-1790.

[S] T. Sauer, Unstable reflexive sheaves on $\mathbf{P}^{3}$, Trans. Amer. Math. Soc., 281 (1984), 633-655.

Received December 20, 1989.

UNIVERSITÀ DI TORINO

via Carlo Alberto, 10

10121 TORINO, ITALY

AND

POLITECNICO DI TORINO

Corso DuCA DEgli AbruzzI, 24

10129 TORINO, ITALY 



\section{PACIFIC JOURNAL OF MATHEMATICS EDITORS}

\author{
V. S. VARADARAJAN \\ (Managing Editor) \\ University of California \\ Los Angeles, CA 90024-1555-05 \\ Herbert Clemens \\ University of Utah \\ Salt Lake City, UT 84112 \\ THOMAS ENRIGHT \\ University of California, San Diego \\ La Jolla, CA 92093
}

R. FINN

Stanford University

Stanford, CA 94305

HeRmann FlaschKa

University of Arizona

Tucson, AZ 85721

VAUGHAN F. R. JoNES

University of California

Berkeley, CA 94720

SteVen KeRCKHOFF

Stanford University

Stanford, CA 94305
C. C. MOORE

University of California

Berkeley, CA 94720

MaRTin ScharLemanN

University of California

Santa Barbara, CA 93106

HAROLd STARK

University of California, San Diego

La Jolla, CA 92093

\section{ASSOCIATE EDITORS \\ R. ARENS \\ E. F. BECKENBACH (1906-1982) \\ B. H. NeumanN \\ F. WoLf \\ (1904-1989) \\ K. YoshidA \\ SUPPORTING INSTITUTIONS \\ UNIVERSITY OF ARIZONA \\ UNIVERSITY OF BRITISH COLUMBIA \\ CALIFORNIA INSTITUTE OF TECHNOLOGY \\ UNIVERSITY OF CALIFORNIA \\ MONTANA STATE UNIVERSITY \\ UNIVERSITY OF NEVADA, RENO \\ NEW MEXICO STATE UNIVERSITY OREGON STATE UNIVERSITY \\ UNIVERSITY OF OREGON \\ UNIVERSITY OF SOUTHERN CALIFORNIA \\ STANFORD UNIVERSITY \\ UNIVERSITY OF HAWAII \\ UNIVERSITY OF TOKYO \\ UNIVERSITY OF UTAH \\ WASHINGTON STATE UNIVERSITY UNIVERSITY OF WASHINGTON}

The Supporting Institutions listed above contribute to the cost of publication of this Journal, but they are not owners or publishers and have no responsibility for its content or policies.

Mathematical papers intended for publication in the Pacific Journal of Mathematics should be in typed form or offset-reproduced (not dittoed), double spaced with large margins. Please do not use built up fractions in the text of the manuscript. However, you may use them in the displayed equations. Underline Greek letters in red, German in green, and script in blue. The first paragraph must be capable of being used separately as a synopsis of the entire paper. In particular it should contain no bibliographic references. Please propose a heading for the odd numbered pages of less than 35 characters. Manuscripts, in triplicate, may be sent to any one of the editors. Please classify according to the 1991 Mathematics Subject Classification scheme which can be found in the December index volumes of Mathematical Reviews. Supply name and address of author to whom proofs should be sent. All other communications should be addressed to the managing editor, or Elaine Barth, University of California, Los Angeles, California 90024-1555-05.

There are page-charges associated with articles appearing in the Pacific Journal of Mathematics. These charges are expected to be paid by the author's University, Government Agency or Company. If the author or authors do not have access to such Institutional support these charges are waived. Single authors will receive 50 free reprints; joint authors will receive a total of 100 free reprints. Additional copies may be obtained at cost in multiples of 50 .

The Pacific Journal of Mathematics (ISSN 0030-8730) is published monthly except for July and August. Regular subscription rate: $\$ 190.00$ a year (10 issues). Special rate: $\$ 95.00$ a year to individual members of supporting institutions.

Subscriptions, orders for numbers issued in the last three calendar years, and changes of address should be sent to Pacific Journal of Mathematics, P.O. Box 969, Carmel Valley, CA 93924, U.S.A. Old back numbers obtainable from Kraus Periodicals Co., Route 100, Millwood, NY 10546.

The Pacific Journal of Mathematics at P.O. Box 969, Carmel Valley, CA 93924 (ISSN 0030-8730) is published monthly except for July and August. Second-class postage paid at Carmel Valley, California 93924, and additional mailing offices. Postmaster: send address changes to Pacific Journal of Mathematics, P.O. Box 969, Carmel Valley, CA 93924.

PUBLISHED BY PACIFIC JOURNAL OF MATHEMATICS, A NON-PROFIT CORPORATION Copyright (C) 1991 by Pacific Journal of Mathematics 


\section{Pacific Journal of Mathematics}

Vol. 150, No. $2 \quad$ October, 1991

Selman Akbulut and Henry Churchill King, Rational structures on

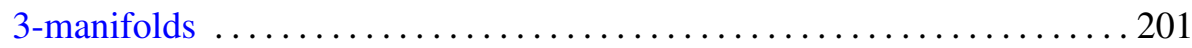

Mark Baker, On coverings of figure eight knot surgeries $\ldots \ldots \ldots \ldots \ldots 215$

Christopher Michael Brislawn, Traceable integral kernels on countably generated measure spaces . ...........................229

William Chin, Crossed products and generalized inner actions of Hopf algebras

Tadeusz Figiel, William Buhmann Johnson and Gideon Schechtman, Factorizations of natural embeddings of $l_{p}^{n}$ into $L_{r}$. II $\ldots \ldots \ldots \ldots \ldots 261$

David Howard Gluck, Character value estimates for groups of Lie type ... 279

Charn-Huen Kan, Norming vectors of linear operators between $L_{p}$

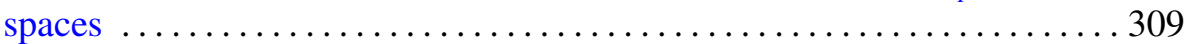

Marko Kranjc, Embedding a 2-complex $K$ in $\mathbb{R}^{4}$ when $H^{2}(K)$ is a cyclic

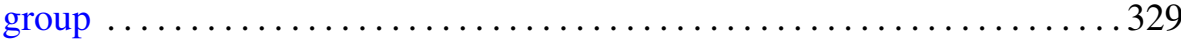

Ka-Lam Kueh, The remainder terms aspect of the theory of the Riemann

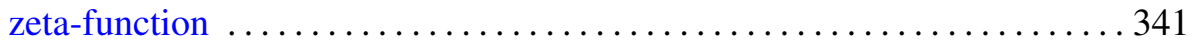

J. A. Marti, Sur la rigidité comparée de fonctions, distributions, ou hyperfonctions analytiques par rapport à un groupe de variables

Margherita Roggero and Paolo Valabrega, Chern classes and cohomology for rank 2 reflexive sheaves on $\mathbf{P}^{3}$ 